Draft Version March 8, 2017

Preprint typeset using $\mathrm{AT}_{\mathrm{E} X} \mathrm{X}$ style emulateapj v. 12/16/11

\title{
PREDICTED EXTENSION OF THE SAGITTARIUS STREAM TO THE MILKY WAY VIRIAL RADIUS
}

\author{
Marion I. P. Dierickx ${ }^{1}$ And Abraham Loeb ${ }^{1}$ \\ Draft version March 8, 2017
}

\begin{abstract}
The extensive span of the Sagittarius (Sgr) stream makes it a promising tool for studying the Milky Way gravitational potential. Characterizing its stellar kinematics can constrain halo properties and provide a benchmark for the Cold Dark Matter galaxy formation paradigm. Accurate models of the disruption dynamics of the Sgr progenitor are necessary to employ this tool. Using a combination of analytic modeling and N-body simulations, we build a new model of the Sgr orbit and resulting stellar stream. In contrast to previous models, we simulate the full infall trajectory of the Sgr progenitor from the time it first crossed the Milky Way virial radius 8 Gyr ago. An exploration of the parameter space of initial phase-space conditions yields tight constraints on the angular momentum of the Sgr progenitor. Our best-fit model is the first to reproduce accurately existing data on the 3D positions and radial velocities of the debris detected $100 \mathrm{kpc}$ away in the $\mathrm{MW}$ halo. In addition to replicating the mapped stream, the simulation also predicts the existence of several arms of the Sgr stream extending to hundreds of kiloparsecs. The two most distant stars known in the Milky Way halo coincide with the predicted structure. Additional stars in the newly predicted arms can be found with future data from the Large Synoptic Survey Telescope. Detecting a statistical sample of stars in the most distant Sgr arms would provide an opportunity to constrain the Milky Way potential out to unprecedented Galactocentric radii.
\end{abstract}

\section{INTRODUCTION}

The Local Group provides a natural laboratory for near-field cosmology and the study of galaxy formation. Dwarf satellites within the Local Group can be characterized at a high level of detail, providing a rich dataset to answer open questions about the structure of dark matter haloes. With a Galactocentric distance of only $\sim 25 \mathrm{kpc}$, the Sagittarius (Sgr) dwarf spheroidal is one of the nearest dwarf galaxies (Kunder \& Chaboyer 2009). First discovered by Ibata et al. (1994), Sgr is in a near polar orbit around the Milky Way (MW) and has experienced multiple passages through the disk (e.g. Law \& Majewski 2010, Purcell et al. 2011). The resulting stream of tidally stripped stars wraps a full $360^{\circ}$ around the celestial sphere. Coincidentally, the Sun's location is close enough to the Sgr orbital plane to likely lie within the width of the debris trail (Majewski et al. 2003).

Starting with Johnston et al. (1995), many studies have attempted to constrain both the properties of Sgr and the MW based on the Sgr debris system. Building on increasingly detailed surveys of the stellar stream's leading and trailing arms, many investigations use the kinematics of the tidal debris as a diagnostic of the gravitational potential (e.g. Ibata et al. 2001; Helmi 2004 Law et al. 2005; Belokurov et al. 2006; Peñarrubia et al. 2006; Law \& Majewski 2010; Deg \& Widrow 2013: Vera-Ciro \& Helmi 2013; Price-Whelan \& Johnston 2013 Sohn et al. 2015). This approach has yielded ambiguous results (1bata et al. 2013), alternatively pointing to prolate (Helmi 2004), oblate (Johnston et al. 2005), spherical (Ibata et al. 2001; Fellhauer at al. 2006), or triaxial (Law et al. 2009; Law \& Majewski 2010 Deg \& Widrow 2013) halo shapes.

\footnotetext{
${ }^{1}$ Astronomy Department, Harvard University, 60 Garden Street, Cambridge, MA 02138, USA; mdierickx@cfa.harvard.edu, aloeb@cfa.harvard.edu
}

Reconstructing the Sgr orbital history consistently with the observed stream necessarily underlies any inferences made about the MW's potential. However, as demonstrated by Jiang \& Binney (2000), different families of orbital histories are allowed depending on the mass of the Sgr progenitor, due to dynamical friction. A more massive Sgr progenitor $\left(\sim 10^{11} \mathrm{M}_{\odot}\right)$ would fall in from larger Galactocentric distances $(\gtrsim 200 \mathrm{kpc})$ and undergo stronger mass stripping and dynamical friction. Conversely, the Sgr progenitor may have been as light as $\sim 10^{9} \mathrm{M}_{\odot}$ provided its initial separation was comparable to current apocentric distances of $\sim 60 \mathrm{kpc}$. The uncertainty in the Sgr progenitor structure therefore goes hand in hand with the uncertainty in its orbital history. Properties of the Sgr dwarf's baryonic components, such as disk rotation, have also been shown to affect features of the resulting tidal stream (Peñarrubia et al. 2010 ).

Recently, the two most widely referenced orbital models for Sgr have been those of Law \& Majewski (2010) and Purcell et al. (2011). The orbit introduced by Purcell et al. (2011) and used by Gòmez et al. (2015) starts with the Sgr progenitor only $80 \mathrm{kpc}$ away from the Galactic center, well within the virial radius of the MW. Earlier phases of the orbit are not simulated and the Sgr progenitor is artificially truncated at its instantaneous Jacobi radius in order to mimic tidal stripping during the early infall stage. However, the initial phase-space coordinates of the progenitor at $80 \mathrm{kpc}$ are taken from Sgr example orbits by Keselman et al. (2009), and not directly based on observable quantities. Therefore the resulting trajectory shares qualitative properties with the true Sgr orbit, but is unlikely to be an accurate match.

The detailed work of Law \& Majewski (2010) integrates the orbit of a test particle from Sgr's current location back in time in a fixed MW-like potential, under the assumption that Sgr is currently moving towards the galactic plane. This model does not utilize the proper 
motion from precision Hubble Space Telescope astrometry (Dinescu et al. 2005; Pryor et al. 2010; Massari et al. 2013). The proper motion predicted by the Law \& Majewski (2010) model is within $2 \sigma$ of the estimates by Dinescu et al. (2005) and Pryor et al. (2010), but better accuracy could likely be achieved by basing the model directly on the transverse velocity measurements. Importantly, inferring the orbit from evolving a test particle backwards in time does not capture tidal stripping effects on the progenitor halo. This approach is valid in the low-mass, low dynamical friction, low stripping regime outlined by Jiang \& Binney (2000), but cannot recover earlier infall phases for a progenitor with mass $\geq 10^{9} \mathrm{M}_{\odot}$. The initial mass of Sgr used by Law \& Majewski $(2010)$ is $6.4 \times 10^{8} \mathrm{M}_{\odot}$, distinctly in the regime where dynamical friction is unimportant.

Since these early efforts to model the Sgr orbit, there has been mounting evidence in favor of a more massive Sgr progenitor (Niederste-Ostholt et al. 2010, Conroy \& Wechsler 2009; Behroozi et al. 2010; Gibbons et al. 2016). This calls for a renewed effort to model the Sgr orbit, simultaneously accounting for higher progenitor mass and initial separation. In this paper, we set out to find a model for the full infall of the Sgr dwarf into the MW, until it reaches its current observed position and velocity. This includes the early infall phase at Galactocentric radii $>60-80 \mathrm{kpc}$ not simulated by Law \& Majewski (2010), Purcell et al. (2011) or Gòmez et al. (2015). The survival of the Sgr satellite until the present day implies that it cannot have formed deep inside the MW halo, where it would have been cannibalized already. Rather, hierarchical models of galaxy formation suggest that the Sgr dwarf likely formed early on in the periphery of the assembling host halo. We therefore aim to initiate the Sgr progenitor at the MW's virial radius at redshift of $z=1$, approximately $8 \mathrm{Gyr}$ ago. The higher initial separation and Sgr mass preclude integrating orbits backwards in time and call for full forward modeling, including tidal stripping and dynamical friction. We therefore use a combination of analytic and N-body modeling in a dual approach to test possible trajectories for Sgr. The high computational cost of full N-body simulations, where both the MW and Sgr progenitor are modeled with live haloes, prohibits a brute force exploration of the parameter space. Therefore, we first carry out a systematic search of the parameter space with a fast semi-analytic, point particle-like model in Section 2. We then perform N-body simulations of the best-fit models with GADGET for comparison with the Sgr tidal stream in Section 3. Finally, we discuss our main conclusions in Section 4.

\section{SEMI-ANALYTIC MODEL}

\subsection{Galaxy Parameters}

Galaxy formation is a continuous process and as a result, the formation redshift of galaxies such as the MW and Sgr dwarf cannot be pinpointed exactly. We aim to trace the system's history out to a redshift of $z=1$, corresponding to approximately 8 Gyr of lookback time. This choice is based on the age of the M-giants in the stream, estimated by Bellazzini et al. (2006) to be $8.0 \pm 1.5$ Gyr. We adopt this evolutionary timescale as the guiding principle for our initial conditions, postulat-
TABLE 1

Galaxy Parameters for Semi-Analytic and N-Body Simulations.

\begin{tabular}{llll}
\hline \hline Parameter & Description & MW & Sgr dSph \\
\hline$M_{\text {NFW }}$ & NFW halo mass & $1 \times 10^{12} \mathrm{M}_{\odot}$ & $1 \times 10^{10} \mathrm{M}_{\odot}$ \\
$c$ & NFW halo concentration & 10 & 8 \\
$M_{\text {halo }}$ & Hernquist total mass & $1.25 \times 10^{12} \mathrm{M}_{\odot}$ & $1.3 \times 10^{10} \mathrm{M}_{\odot}$ \\
& Particles in halo & $1.16 \times 10^{6}$ & $1.17 \times 10^{4}$ \\
$R_{200 c, z=0}$ & Present-day virial radius & $206 \mathrm{kpc}$ & $44 \mathrm{kpc}$ \\
$r_{\mathrm{H}}$ & Hernquist scale radius & $38.35 \mathrm{kpc}$ & $9.81 \mathrm{kpc}$ \\
$M_{\text {disk }}$ & Disk mass & $0.065 M_{\text {halo }}$ & $0.06 M_{\text {halo }}$ \\
& Particles in disk & $2.03 \times 10^{6}$ & $1.95 \times 10^{4}$ \\
$M_{\text {bulge }}$ & Bulge mass & $0.01 M_{\text {halo }}$ & $0.04 M_{\text {halo }}$ \\
& Particles in bulge & $3.125 \times 10^{5}$ & $1.3 \times 10^{4}$ \\
$b_{0}$ & Disk scale length & $3.5 \mathrm{kpc}$ & $0.85 \mathrm{kpc}$ \\
$c_{0}$ & Disk scale height & $0.15 b_{0}$ & $0.15 b_{0}$ \\
$a$ & Bulge scale length & $0.2 b_{0}$ & $0.2 b_{0}$ \\
\hline \hline
\end{tabular}

Note. - MW parameters are adapted from Gòmez et al. (2015). The parameters for Sgr are determined based on several considerations: the virial mass is chosen as estimated by Niederste-Ostholt et al. (2010), the disk scale radius following Gòmez et al. (2015), and a stellar mass percentage larger than in Gòmez et al. (2015) by a factor of 3 for practical (resolution) reasons. We use standard $\Lambda$ CDM cosmological parameters values of $H_{0}=$ $70 \mathrm{~km} \mathrm{~s}^{-1} \mathrm{Mpc}^{-1}$ and $\Omega_{\mathrm{m}}=0.27$ (Planck Collaboration 2015).

ing that the Sgr progenitor may first have crossed the MW's virial radius around that time. We adopt a fiducial virial mass of $10^{12} \mathrm{M}_{\odot}$ for the $\mathrm{MW}$, consistent with recent estimates (e.g. Xue et al. 2008). Various simulations of halo assembly histories suggest that today's MW-like haloes would have built up at least half of their mass by $z=1$, with a significant scatter of $\sim 2 \times 10^{11} \mathrm{M}_{\odot}$ depending on the halo's accretion history (e.g. Torrey et al. 2015, Lu et al. 2016). Using the spherically symmetric top-hat framework of halo formation, the virial radius of a $5 \times 10^{11} \mathrm{M}_{\odot}$ galaxy collapsing at $z=1$ is approximately $124 \mathrm{kpc}$ (Barkana \& Loeb 2001). We therefore initiate the Sgr progenitor at a Galactocentric radius of $125 \mathrm{kpc}$ and aim to trace its evolution over a period of 8 Gyr.

Halo growth is believed to occur inside-out, with later additions of mass being appended on the outskirts of the halo (e.g. Loeb \& Peebles 2003). This picture has been validated in simulations (e.g. Wellons et al. 2016) and observations (e.g. de la Rosa et al. 2016) of highredshift massive compact galaxies. Cosmological simulations also show that after $z=1$, most MW analogs do not undergo a major merger (e.g. Fakhouri et al. 2010) and have inner halo profiles that remain essentially fixed (e.g. Gao et al. 2004, Mollitor et al. 2015). Studies of the stellar populations in the MW disk suggest a quiet evolution since $z=2$, with no significant mergers in the last $\sim 10$ Gyr (Wyse 2001; Hammer et al. 2007). The Sgr orbit in our model is contained inside Galactocentric radii $<125 \mathrm{kpc}$ and is consequently not impacted by the later addition of matter outside of this sphere (assuming spherical symmetry). As a result, we maintain the same initial halo profile throughout each simulation. A halo's scale radius $r_{s}$ is related to its virial radius $R_{\text {vir }}$ and concentration parameter $c$ by the relationship $r_{s}=R_{\mathrm{vir}} / c$. As the halo accretes mass on its outskirts, the scale radius is maintained constant while the virial radius and the concentration parameter grow. Following the concentration growth of galaxies in the models of Diemer \& Kravtsov (2015), a MW analog would have grown from 
$c \simeq 7, R_{\text {vir }} \simeq 125 \mathrm{kpc}$ at $z=1$ to $c \simeq 10, R_{\mathrm{vir}} \simeq 200 \mathrm{kpc}$ at $z=0$, giving a nearly constant scale radius of $\sim 18$ $20 \mathrm{kpc}$. For a $z=0 \mathrm{MW}$ analog (with parameters as outlined in Table 1), the mass inside a radius of $125 \mathrm{kpc}$ is approximately $7 \times 10^{11} \mathrm{M}_{\odot}$, consistent with the virial mass of $(5 \pm 2) \times 10^{11} \mathrm{M}_{\odot}$ of its $z=1$ progenitor. Beyond the prescriptions for the MW's evolution adopted here, Peñarrubia et al. (2006) provide an in-depth study of modeling tidal streams in evolving dark matter halos. Their simulations suggest that the debris configuration reflects the present galactic potential shape rather than its evolution. Our choice of a constant host potential profile throughout the orbit of Sgr is therefore unlikely to have a strong effect on the phase-space distribution of the stream.

The MW potential includes three components: a dark matter halo and bulge following Hernquist profiles (Hernquist 1990), and an exponential disk:

$$
\Phi_{\mathrm{MW}}=\Phi_{\text {halo }}+\Phi_{\text {disk }}+\Phi_{\text {bulge }}
$$

where

$$
\begin{aligned}
\Phi_{\text {halo }}(r) & =-\frac{G M_{\text {halo }}}{r+r_{\mathrm{H}}}, \\
\Phi_{\text {disk }}(r) & =-G M_{\text {disk }}\left(\frac{1-e^{-r / b_{0}}}{r}\right), \\
\Phi_{\text {bulge }}(r) & =-\frac{G M_{\text {bulge }}}{r+c_{0}} .
\end{aligned}
$$

For simplicity, the disk and bulge components are omitted for Sgr in the semi-analytic model, but included in the full simulation described in Section 3. The parameters of both galactic potentials are summarized in Table 1. The parameters of the Hernquist halo potentials are chosen such that the mass enclosed within the radius of interest matches that of fiducial Navarro, Frenk \& White profiles (NFW; Navarro et al. 1997) profiles. The resulting enclosed mass curves are shown in Figure 1. For the MW, we adjust the enclosed mass inside the initial distance between the galaxy centers, $d_{\text {init }}=125 \mathrm{kpc}$. A total halo mass of $M_{\text {halo }}=1.25 \times 10^{12} \mathrm{M}_{\odot}$ and a scale radius of $r_{\mathrm{H}}=38.35 \mathrm{kpc}$ matches the fiducial NFW halo with a virial mass $M_{\mathrm{MW}}=10^{12} \mathrm{M}_{\odot}$ and a concentration parameter of 10 . For Sgr, we use $M_{\text {halo }}=1.3 \times 10^{10} \mathrm{M}_{\odot}$ and $r_{\mathrm{H}}=9.81 \mathrm{kpc}$. This corresponds to the mass enclosed within the initial tidal radius, $r_{\text {tidal, init }} \simeq 25 \mathrm{kpc}$, of an NFW potential with $M_{\mathrm{Sgr}}=10^{10} \mathrm{M}_{\odot}$ and $c_{\mathrm{Sgr}}=$ 8.

Our choice of initial parameters for Sgr is based on the study by Niederste-Ostholt et al. (2010), who reconstruct the properties of the progenitor by conducting a census of the stellar tidal debris. A lower bound of $(9.6-13.2) \times 10^{7} \mathrm{~L}_{\odot}$ is inferred for the progenitor's luminosity by summing up the luminosities of the Sgr core, leading and trailing arms. Relating this value to results from cosmological $N$-body simulations, NiedersteOstholt et al. (2010) estimate a mass of $\sim 10^{10} \mathrm{M}_{\odot}$ for the Sgr dark matter halo prior to tidal disruption. Based on this choice of progenitor mass, the mass-concentration relation models of Diemer \& Kravtsov (2015) suggest a low concentration of $c \simeq 8$ at $z=1$. We then use these parameters as our reference NFW profile, and tune the Hernquist profile parameters to match the mass enclosed inside the initial tidal radius of the system (see Figure 1). The resulting Hernquist scale radius $r_{\mathrm{H}}=9.81 \mathrm{kpc}$ is consistent with the values used by Gòmez et al. (2015) for their 'Light Sgr' model, which has a mass of $3.2 \times 10^{10} \mathrm{M}_{\odot}$. Since only one wrap of the debris is considered and additional luminous matter may be located at apocenter pile-ups, the true Sgr progenitor mass may exceed the lower bound established by Niederste-Ostholt et al. (2010). Given a total luminosity of $\sim 10^{8} \mathrm{~L}_{\odot}$, cosmological abundance matching would suggest a higher mass of $\sim 10^{10.5}-10^{11} \mathrm{M}_{\odot}$ (Conroy \& Wechsler 2009, Behroozi et al. 2010). Exploring a range of different progenitor models, Gibbons et al. (2016) find that masses $\gtrsim 6 \times 10^{10} \mathrm{M}_{\odot}$ are most consistent with the velocity dispersion of the stream stellar populations. Still, the orbit presented here is the first physically motivated model for the Sgr infall that includes a progenitor mass closer to the most recent mass estimates.

\subsection{Methodology}

In our search for an orbital model for the Sgr dwarf spheroidal, we start testing possible trajectories with an approximate point-particle approach. From predetermined initial conditions, the equations of motion are solved numerically forward in time with a fourth order Runge-Kutta method. Both the dynamics of Sgr in the MW potential and the MW's response to the gravitational attraction of Sgr are modeled, including tidal stripping and dynamical friction for Sgr. We emphasize the fact that the MW potential is not held fixed at the origin; rather, we account for the mutual gravitational attraction between the MW and Sgr and allow the MW to move freely about the common center of mass of the system. As the studies of Dierickx et al. (2014) and Gòmez et al. (2015) have shown, the response of a MW-like host galaxy to an infalling satellite can be significant. This is particularly relevant in the high separation, high Sgr progenitor mass regime explored here.

Keeping the initial separation fixed, the remaining free parameters quantify the amount of initial orbital angular momentum we grant Sgr at its starting location. We specify two quantities: $v_{\text {init }}$, the magnitude of the Sgr velocity, and $\theta_{\text {init }}$, the angle between the velocity vector and the direction to the MW center (see Figure 2). We integrate a two-dimensional grid of 2500 orbits with $v_{\text {init }}$ ranging from 0 to $\sim 320 \mathrm{~km} \mathrm{~s}^{-1}$, the NFW escape velocity from the MW halo at the initial radius of the Sgr progenitor, and $\theta_{\text {init }}$ ranging from 10 to 90 degrees. With total energy per unit mass defined as $E=\frac{1}{2} v_{\text {init }}^{2}-\Phi\left(d_{\text {init }}\right)$, the upper limit on velocity investigated here corresponds to $E=0$, i.e. a marginally bound Sgr falling into the MW from a larger distance. We specify a nonzero lower limit on the initial angle in order to avoid purely radial orbits, which cause pathological behavior in the model. Angles $>90^{\circ}$ are excluded as they would imply a Sgr progenitor velocity directed away from the MW.

Throughout the orbital integration, the MW experiences acceleration from a standard Hernquist gravitational potential for a tidally-truncated Sgr. For the Sgr satellite however, the following acceleration is calculated at Galactocentric coordinates $\vec{r}$ :

$$
\vec{a}=-\frac{G M_{\text {halo }}(<r)}{r^{3}} \vec{r}-\nabla \Phi_{\text {disk }}-\nabla \Phi_{\text {bulge }}+\vec{a}_{\mathrm{DF}},
$$



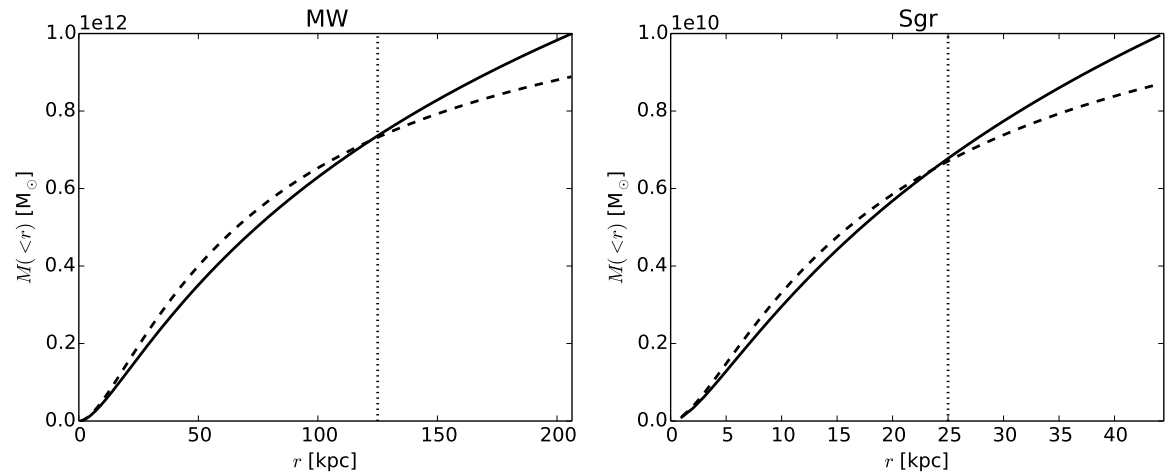

FIG. 1. - Enclosed mass profiles for the chosen halo profiles of the MW and Sgr. The solid line shows the reference NFW profile (Navarro et al. 1997) for each galaxy. Dotted lines indicate the boundary radii where the enclosed mass values are matched: $d_{\text {init }}=125 \mathrm{kpc}$ for the MW, and $r_{\text {tidal, init }} \simeq 25 \mathrm{kpc}$ for Sgr. The dashed lines show the resulting Hernquist approximations to the NFW profiles. Profile parameters are provided in Table 1 .

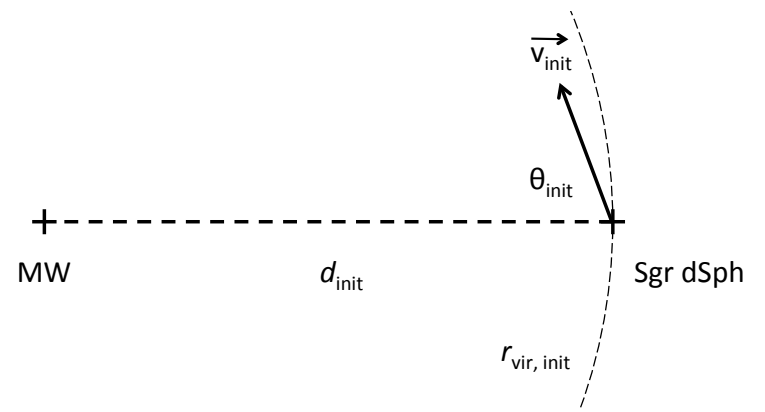

FIG. 2.- The initial configuration of the MW and Sgr dSph progenitor in the semi-analytic orbital model. Sgr is initially located a distance $d_{\text {init }}$ away from the center of the Galaxy, corresponding to the virial radius of the MW at that time $(z \sim 1)$. Sgr is given an initial velocity vector $\vec{v}_{\text {init }}$ with variable magnitude and angle $\theta_{\text {init }}$.

where $M_{\text {halo }}(<r)$ is the mass interior to radius $r$ of the host halo, given simply by $M_{\text {halo }}(<r)=M_{\text {halo }}\left[r^{2} /(r+\right.$ $\left.r_{H}\right)^{2}$ ] (Hernquist 1990). The acceleration introduced by dynamical friction, $\vec{a}_{\mathrm{DF}}$, is modeled using the Chandrasekhar formula (Binney \& Tremaine 1987, eq. 7.18):

$$
\begin{aligned}
\vec{a}_{\mathrm{DF}}=-\frac{4 \pi \ln (\Lambda) G^{2} \rho(r) M_{\mathrm{Sgr}}\left(<r_{t}\right)}{v^{3}} \times & \\
& {\left[\operatorname{erf}(X)-\frac{2 X}{\sqrt{\pi}} \exp \left(-X^{2}\right)\right] \vec{v} }
\end{aligned}
$$

where $\rho(r)$ is the density of the host halo at Galactocentric distance $r, M_{\mathrm{Sgr}}\left(<r_{t}\right)$ is the Sgr mass interior to its minimum tidal radius $r_{t}$, and $\ln (\Lambda)$ is the Coulomb logarithm. The remaining term involves $X=v / \sqrt{2} \sigma$, where $v$ is the satellite velocity and $\sigma$ is the one-dimensional velocity dispersion of particles in the host halo (given by Hernquist 1990, eq. 10). Based on the formalism used by Besla et al. (2007) in their study of the orbital evolution of the Magellanic Clouds, we adopt an alternative timedependent Coulomb logarithm as follows (Hashimoto et al. 2003):

$$
\ln (\Lambda)=\ln \left(\frac{r}{1.4 \epsilon}\right)
$$

where $\epsilon$ is a softening length variable used by Hashimoto et al. (2003) to model the Large Magellanic Cloud (LMC) with a Plummer sphere. This time-dependent parameterization of the Coulomb logarithm is also in agreement with the calibration carried out by van der Marel et al. (2012b) for the M31-M33 interaction (which is of similarly unequal mass). We ran a series of semi-analytic orbits to test the friction parameterization, and find that setting $\epsilon \simeq 1 \mathrm{kpc}$ gives the best agreement with $N$-body integrations of the same initial conditions. This halo parameter is smaller by a factor of 3 compared to the parameterization used by Hashimoto et al. (2003) for the LMC, not surprising given that the mass ratio between host and satellite is more unequal by a factor of 10 in our case. Comparing to the standard formalism where $\Lambda=b_{\max } / b_{\min }$ (Binney \& Tremaine 1987), we have in essence set the impact parameter $b_{\min }$ to $\sim 1.4 \mathrm{kpc}$ (a reasonable value given the large range of initial conditions explored here) and introduced a time dependence for the cutoff radius $b_{\max }$. These adjustments are generally expected to have a minor effect given their logarithmic contribution to the dynamical friction exerted on Sgr.

Sgr experiences tidal forces as it moves across the MW halo, leading to the outermost layers of material being stripped. The instantaneous tidal radius for Sgr, $r_{t}$, is found by solving the following equation numerically (King 1962):

$$
r_{t}=r\left[\frac{1}{2} \frac{M_{\mathrm{Sgr}}\left(<r_{t}\right)}{M_{\mathrm{MW}}(<r)}\right]^{1 / 3}
$$

Since material that has been stripped is considered lost, the tidal radius is computed at each time step and if necessary, updated to the lowest value calculated so far. 
Using this prescription we model dynamical friction on a progressively less massive Sgr halo. Since the lost Sgr mass is negligible compared to the MW mass, we ignore its contribution to the MW potential.

From its initial location at $d_{\text {init }}=125 \mathrm{kpc}$ with varying velocity vector $\vec{v}_{\text {init }}$, the orbit of the Sgr progenitor is integrated forward in time for 10 Gyr with 10 Myr time steps. The current position of Sgr can be described by its galactic longitude and latitude $(l, b)=\left(5.6^{\circ},-14.2^{\circ}\right)$ (Majewski et al. 2003) and its heliocentric distance $d_{\text {helio }}=25 \pm 2 \mathrm{kpc}$ (Kunder \& Chaboyer 2009). We define a right-handed Cartesian coordinate system centered on the Galactic Center, with the $z$-axis pointing towards the North Galactic Pole and with the Sun's current location at $[-8,0,0] \mathrm{kpc}$ (Honma et al. 2012; Reid et al. 2014). The coordinates of Sgr in this system can then be found with the following simple transformations (in units of kiloparsecs):

$$
\begin{aligned}
& x=d_{\text {helio }} \cos b \cos l-8, \\
& y=d_{\text {helio }} \cos b \sin l, \\
& z=d \sin b .
\end{aligned}
$$

Applying these equations, the current position vector of the center of Sgr is $\vec{r}_{\mathrm{Sgr}}$, obs $=(16.1,2.35,-6.12) \mathrm{kpc}$, in agreement with the values provided e.g. by Law \& Majewski (2010). The heliocentric radial velocity of Sgr has been measured at $140 \pm 0.33 \mathrm{~km} \mathrm{~s}^{-1}$ (weighted mean of the Sgr,N and M54 average velocities estimates in Table 5 of Bellazzini et al. 2008), and its proper motion in the equatorial coordinate system is $\left(\mu_{\alpha}, \mu_{\delta}\right)=$ $(-2.95 \pm 0.18,-1.19 \pm 0.16)$ mas $\mathrm{yr}^{-1}$ (Massari et al. 2013). Using these heliocentric velocity components, the Cartesian, right-handed galactic space velocity $(U, V, W)$ is calculated following Johnson \& Soderblom (1987). Finally, these heliocentric space velocities are converted to the Galactic Rest Frame (GSR) by adding contributions from the local standard of rest and solar peculiar motions (Schönrich et al. 2010, Reid et al. 2014):

$\vec{v}_{\mathrm{GSR}}\left[\mathrm{km} \mathrm{s}^{-1}\right]=(U, V, W)+(0,237,0)+(11.1,12.24,7.25)$,

This yields a current GSR velocity vector for Sgr of $\vec{v}_{\text {Sgr, obs }}=(242.5,5.6,228.1) \mathrm{km} \mathrm{s}^{-1}$ and a total velocity magnitude of $333 \pm 30 \mathrm{~km} \mathrm{~s}^{-1}$.

At every time step along the trajectory, the quality of the match to the present-day configuration is investigated. This is a first done in a spherically symmetric sense before finding a best-match orientation for the Sun's location. At every step, the MW-Sgr distance and the Sgr velocity magnitude in the galaxy rest frame are compared to the observed values outlined above. For the times when Sgr is within $3 \sigma$ of the correct galactocentric distance and the correct velocity magnitude, the analysis progresses to the next step. At this stage, it is necessary to orient the system in order to further quantify the match to observables. We utilize the spherical symmetry of the host potential to identify which point on a $8 \mathrm{kpc}$-radius sphere around the Galactic Center provides the best match to the Sun's location.

To this end, we construct a Fibonacci lattice of 1,600 points with radius $8 \mathrm{kpc}$ centered at the Galactic Center. The Fibonacci lattice is a convenient method to generate any number $N$ of evenly distributed points on the surface of a sphere, with each point representing almost the same area (Gonzàlez 2010). Points are arranged along a tightly wound generative spiral, and using the golden angle $\left(\sim 137.5^{\circ}\right)$ as the value for the longitudinal turn between consecutive points maximizes packing. With $N=1,600$, every lattice point occupies on average $\sim 25 \mathrm{deg}^{2}$, representing a one-dimensional positional uncertainty of approximately $5 \mathrm{deg}$. Scaled by the ratio of the Sgr heliocentric distance to the Sun's galactocentric distance (approximately a factor of 3 ), a lattice with $N=1,600$ points yields an angular uncertainty of $\sim 1.5-2 \mathrm{deg}$ in the position of Sgr in the sky without becoming computationally prohibitive. This is acceptable given the many approximations and the uncertainties associated with the parameters of the galactic potentials.

For every candidate Sun position on the lattice, we compute the following 6 quantities: i) The Galactic Center - Sgr distance; ii) Sgr heliocentric distance; iii) the Galactic Center - Sgr - Sun angle; iv) the Sgr heliocentric radial velocity; v) the Sgr heliocentric transverse velocity magnitude; vi) the angle between the Sgr transverse velocity vector and the direction to the Galactic Center. The match to the corresponding measured numbers is quantified via a chi-squared test. The parameter values and associated errors used in the analysis are provided in Table 2, The lattice point with the lowest reduced chi-squared is identified and recorded as the best-match position for the Sun at each favorable time step in the run. The moment along each trajectory with the lowest reduced chi-squared between 7 and $8.5 \mathrm{Gyr}$ after initialization is extracted in order to compare different sets of initial conditions.

\subsection{Results of Semi-Analytic Modeling}

Figure 3 shows the reduced chi-squared values associated with the best-match snapshots along each trajectory, over the initial parameter space outlined in Section 2.2. In this color map, darker pixels indicate a closer match to the observed position and velocity of Sgr today. No matches are found for initial angles $\theta_{\text {init }} \lesssim 30^{\circ}$, indicating that nearly radial orbits are incompatible with the distance and velocity constraints imposed by the available data for Sgr. At high initial incidence angles $\left(\theta_{\text {init }} \gtrsim 60^{\circ}\right)$, a specific range of initial velocities $\left(80 \mathrm{~km} \mathrm{~s}^{-1} \lesssim v_{\text {init }} \lesssim 100 \mathrm{~km} \mathrm{~s}^{-1}\right)$ is preferred independently of the angle. Tangential velocities in this range are reasonable given analogous measurements of Local Group satellites: the M33 tangential velocity with respect to M31 is $\sim 129 \mathrm{~km} \mathrm{~s}^{-1}$ (van der Marel et al. 2012a); the tangential velocities of the LMC and SMC with respect to the MW are $\sim 314$ and $\sim 61 \mathrm{~km} \mathrm{~s}^{-1}$, respectively (Kallivayalil et al. 2013).

Contours of constant initial angular momentum are superimposed on the color map in Figure 3. Values of reduced chi-squared indicating a good match $\left(\log _{10}\left(\chi_{r}^{2}\right) \lesssim\right.$ $0.5)$ are tightly grouped together in a "valley" of constant angular momentum. This suggests that in order to reach the current observed position and velocity of Sgr, a narrow range of initial angular momenta is preferred. The $v_{\text {init }}$ width of this range per unit mass and distance is approximately $20 \mathrm{~km} \mathrm{~s}^{-1}$. We thus conclude that the model favors a specific value for the angular momentum Sgr had 7-8 Gyr ago, upon crossing the MW virial radius 
TABLE 2

Observable and Simulated Parameters Used in Chi-Squared Analysis.

\begin{tabular}{lcccc}
\hline Quantity & Observed Value & Semi-Analytic Model & $N$-body Model & Observational Error $(\sigma)$ \\
\hline (i) $[\mathrm{kpc}]$ & 17.4 & 18.6 & 21.0 & 2.0 \\
(ii) $[\mathrm{kpc}]$ & 25.0 & 26.0 & 27.6 & 2.0 \\
(iii) $\left[{ }^{\circ}\right]$ & 6.92 & 7.6 & 10.9 & 5 \\
(iv) $\left[\mathrm{km} \mathrm{s}^{-1}\right]$ & 178.8 & 180.8 & 172.8 & 1.5 \\
(v) $\left[\mathrm{km} \mathrm{s}^{-1}\right]$ & 281.0 & 353.8 & 285.0 & 30 \\
(vi) $\left[{ }^{\circ}\right]$ & 162.0 & 160.4 & 162.8 & 1.5 \\
\hline Resulting $\chi_{r}^{2}$ & - & 1.32 & 2.28 & - \\
\hline \hline
\end{tabular}

Nоте. - The six reference quantities used in the $\chi^{2}$ analysis are provided along with the simulated parameters for both the best-match analytic and $N$-body models (see Section 3). The table rows are as follows: (i) GC - Sgr distance [kpc]; (ii) Sgr - Sun distance [kpc]; (iii) GC - Sgr - Sun angle $\left[^{\circ}\right]$; (iv) Sgr heliocentric line of sight velocity $\left[\mathrm{km} \mathrm{s}^{-1}\right]$; (v) Sgr heliocentric transverse velocity [km s$\left.{ }^{-1}\right]$; (vi) Angle between Sgr transverse velocity vector and the direction to the GC [०].

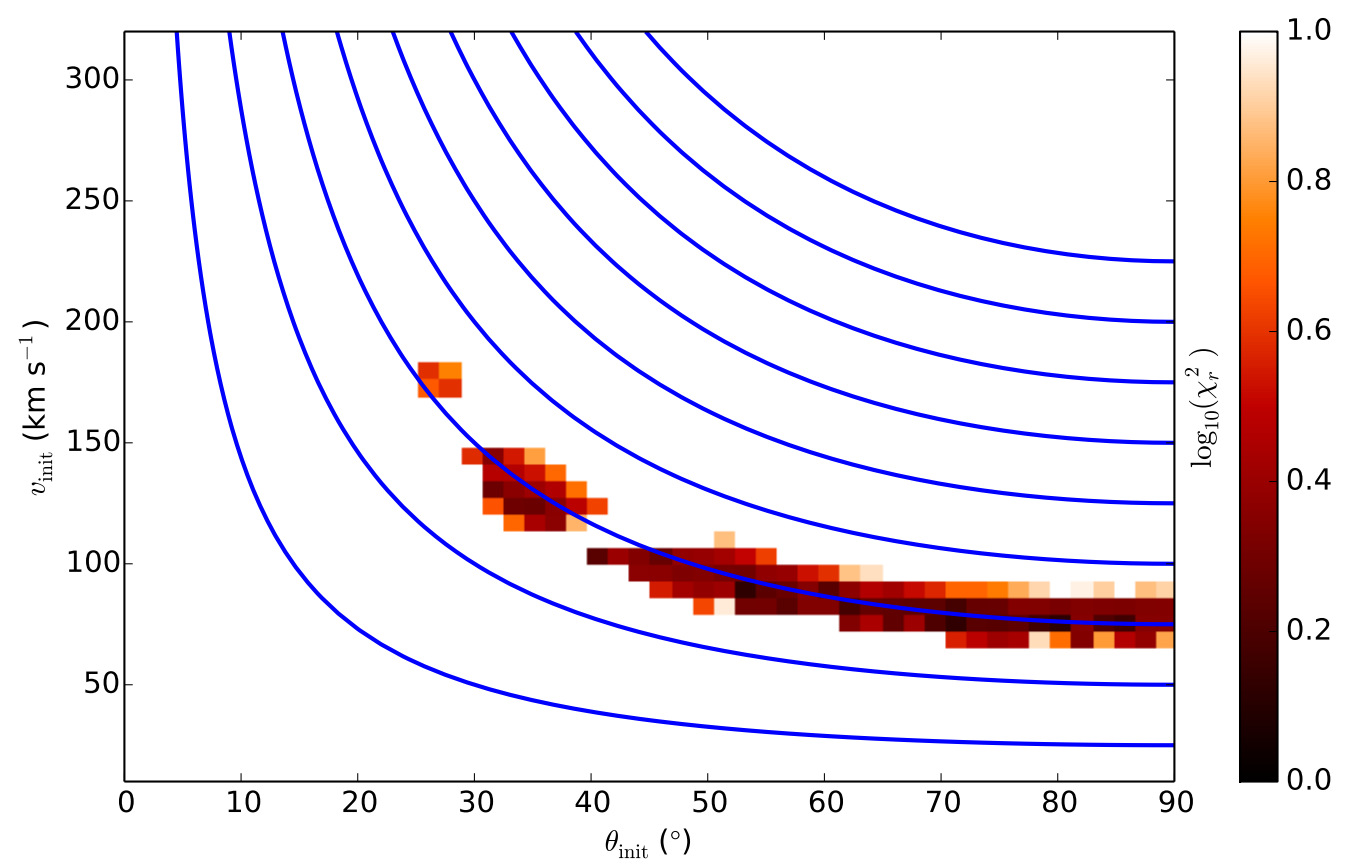

FIG. 3.- Quality of match to the Sgr observed phase-space coordinates over the initial parameter space considered in our semi-analytic model. The angle of the initial velocity vector away from the MW $\left(\theta_{\text {init }}\right)$ is shown on the $x$-axis and the initial velocity magnitude $\left(v_{\text {init }}\right)$ is plotted on the $y$-axis. Values of $\log _{10}\left(\chi_{r}^{2}\right)>1$ are too large to be of interest and are uniformly colored white. Blue lines show contours of constant initial specific angular momentum per unit distance. (These are simply given by $v_{\text {init }} \sin \theta_{\text {init }}=$ constant since the initial MW-Sgr separation and Sgr progenitor mass are kept constant.) The contours range from 25 to $250 \mathrm{~km} \mathrm{~s}^{-1}$ for $\theta_{\text {init }}=90^{\circ}$ and are evenly spaced every $25 \mathrm{~km} \mathrm{~s}^{-1}$.

for the first time.

Next we extract the set of initial parameters with the lowest reduced chi-squared from Figure 3 , and the resulting orbit is shown in Figure 4. In terms of the parameters defined in Figure 2, an initial velocity of $v_{\text {init }}=72.6 \mathrm{~km} \mathrm{~s}^{-1}$ directed at an angle of $\theta_{\text {init }}=80.8^{\circ}$ yields a $\chi_{r}^{2}$ value of 1.32 at a time 7.71 Gyr after the start of the integration (see Table 2). The left panel of Figure 4 shows the resulting trajectory of the Sgr progenitor (in blue) and the MW's drift (in black). The right panel shows the separation between the two galaxies as a function of time (dashed line) as well as the minimum tidal radius of the Sgr galaxy calculated as described in Section 2 (black line). Figure 5 presents a comparison of the best-match simulated and measured phase-space coordinates of Sgr. Overall the semi-analytic model reproduces the position and velocity vector of Sgr very well.

\section{N-BODY SIMULATION}

So far we have only used the phase-space coordinates measured for the Sgr remnant in order to constrain its infall history into the MW. However, additional data available for the Sgr stellar stream from various surveys provide an important way to assess how our model compares to observations. The semi-analytic model described in $\S 2.2$. while useful in exploring parameter space, does not generate mock debris streams. Importantly, we also wish to improve on the spherical symmetry approximation made previously by including a more realistic live potential for the MW. We therefore seek to produce a fiducial model of the Sgr orbit and the associated stream by running $N$-body realizations of the most promising trajectory from the semi-analytic model.

\subsection{Parameters and Initial Conditions}



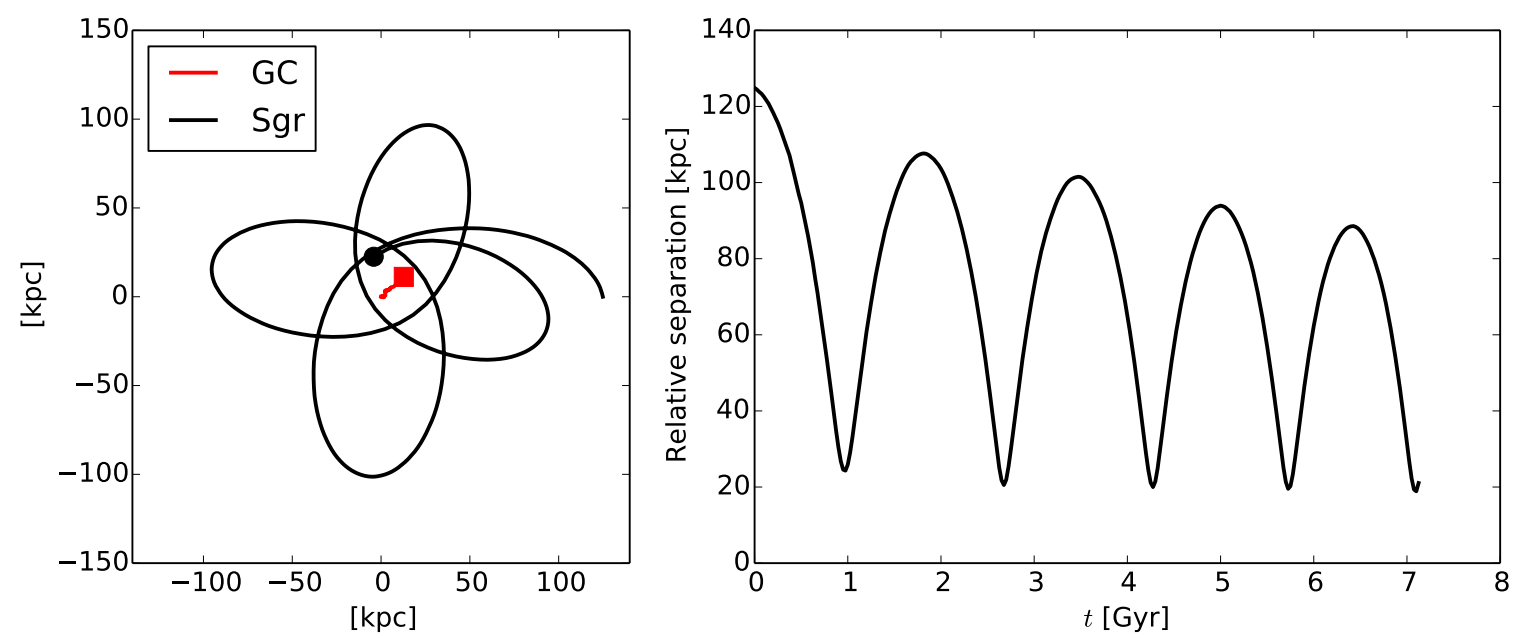

FIG. 4.- Overview of the best-fit Sgr orbit computed by the semi-analytic model described in Section 2 Left panel: trajectories of the Sgr progenitor (red line) and MW Galactic Center (GC; black line) in the Sgr orbital plane. The current locations of the two galaxies are indicated by a colored dot and square, respectively. Right panel: separation between the centers of the two galaxies (solid line) and the minimum tidal radius (dashed line) of Sgr as a function of time since the beginning of the calculation. The closest match to the current position and velocity of Sgr is reached after 7.71 Gyr.
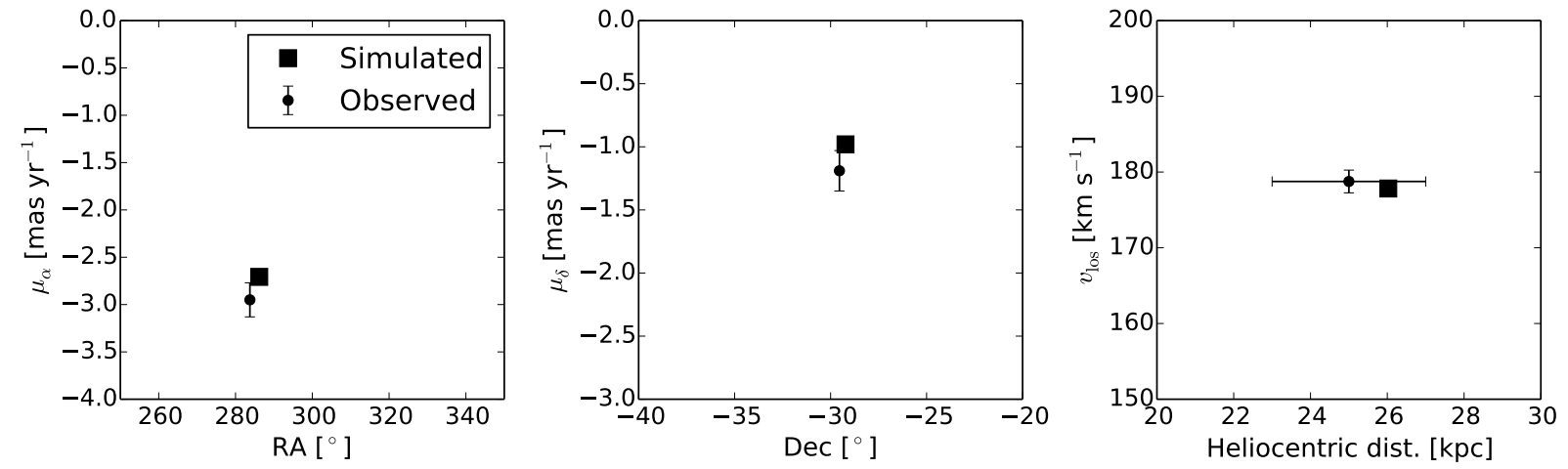

Fig. 5.- Comparison of the observed and simulated heliocentric coordinates of Sgr in the best-fit orbit from the semi-analytic model. The position and velocity are shown as projected on the sky in equatorial coordinates and along the line of sight. Error bars show the $1 \sigma$ uncertainties associated with each measurement. The distance estimate is taken from Kunder \& Chaboyer (2009), the proper motion measurements from Massari et al. (2013), and the line of sight velocity from Bellazzini et al. (2008).

With the simultaneous aims of checking the semianalytic formalism described earlier and generating a mock Sgr stream, we utilize the $N$-body code GADGET (Springel 2005) to re-run the best-fit trajectory presented in Section 2.3 After initially exploiting a simplified spherically symmetric model for the host potential, we now take advantage of having pinned down the best-fit location for the Sun to introduce a flattened disk potential for the MW. With Sgr initialized at (125, $0,0) \mathrm{kpc}$ and with $\vec{v}_{\text {init }} \simeq(-10,0,70) \mathrm{km} \mathrm{s}^{-1}$, the MW disk inclination is defined by the Sun's position at $\sim(7,-1,3) \mathrm{kpc}$. The parameters of the Hernquist halo, bulge and exponential disk components of the simulated host and satellite galaxies are summarized in Table 1 . Since the purpose of this study is to shed light on the dynamical history of Sgr (rather than model its star formation history, for example), the simulation does not include hydrodynamics. The absence of disk gas components should not affect the dynamics of the system, as gas represents only a small percentage of the galaxies' mass budgets.
Full $N$-body simulations of the MW-Sgr interaction are costly because of the uneven ratio in progenitor mass between the two galaxies. As a result, only a few studies so far (e.g. Purcell et al. 2011, 2012, Gòmez et al. 2015) have modeled the MW with a live halo rather than a static potential. However, given the significant mass of the Sgr satellite and its repeated passages at low Galactocentric distances, it is expected to have strong effects on the structure not only of the MW disk, but also lead to significant dark matter overdensities in the halo (Purcell et al. 2012). Both the host and satellite haloes are live in our simulation in order to capture such time-dependent effects on the structure of the MW potential. We aim to resolve the total visible mass of $\operatorname{Sgr}\left(\sim 10^{9} \mathrm{M}_{\odot}\right.$ - see Table 1) to the order of a few tens of thousands of particles in order to sufficiently populate the tidal stream. Therefore, we choose a mass resolution of $4 \times 10^{4} \mathrm{M}_{\odot}$ per stellar particle, yielding 20,800 stellar particles in Sgr. This in turn implies a required $\sim 2.3 \times 10^{6}$ particles to simulate the MW's stellar mass of $\sim 9.4 \times 10^{10} \mathrm{M}_{\odot}$. We use a dark matter particle resolution of $10^{6} \mathrm{M}_{\odot}$, giving $\sim 1.2 \times 10^{6}$ 
and $\sim 1.2 \times 10^{4}$ dark matter particles for the MW and Sgr galaxies respectively. The majority of the computational cost in the simulation therefore comes from adequately resolving the baryonic component of the Sgr dwarf. The option of modeling the MW with fewer particles of higher mass is undesirable, as it leads to rapid two-particle relaxation and introduces artificial disruption of the Sgr satellite (Jiang \& Binney 2000). We use an adaptive time step of maximum length $10 \mathrm{Myr}$, and the softening lengths for the baryonic and dark matter particles are $41 \mathrm{pc}$ and $214 \mathrm{pc}$, respectively.

\subsection{Overview of Simulation}

Figure 6 presents a general overview of the orbit of Sgr computed here. The apocenter and pericenter distances decrease at each passage due to dynamical friction. We note that friction is more efficient in the live simulation, gradually shrinking the apocentric distances reached by the satellite as compared to the semi-analytic calculation. Similarly, the discrepancy between the orbital periods in each model grows in time, such that after 7 Gyr of evolution, the timing of the fourth pericenter passage differs by $\sim 0.6 \mathrm{Gyr}$. In the $N$-body case, the time corresponding to the present-day configuration is reached after 7.13 Gyr. At that time, the match between the observed and simulated phase-space coordinates of Sgr is quantified by a reduced chi-squared value of 2.28 , slightly worse than for the semi-analytic model (see Table 2). Figure 7 shows both the observed and simulated three-dimensional position and velocity of the Sgr core at the present time in relation to the Sun. Across the many test simulations carried out for this work, it is generally the case that the simulated Sgr core is slightly more distant than observed. This challenge in reaching small Galactocentric radii likely arises due to our choice of initial conditions, with the Sgr progenitor starting at larger distances than previously considered for example by Law \& Majewski (2010) and Purcell et al. (2011). Despite the much larger initial separation chosen here, the MW-Sgr distance and relative velocity vectors are still matched within approximately $2 \sigma$. Stronger dynamical friction may help reaching smaller Galactocentric separations, suggesting that the Sgr progenitor may have initially been more massive than considered in this study $\left(M_{\mathrm{Sgr}}=10^{10} \mathrm{M}_{\odot}\right)$. Testing the dependence of the parameter match on the model MW halo could also provide useful constraints on its properties, a possibility we plan to explore in more depth in a follow-up paper.

Figure 8 presents the behavior of the Sgr progenitor's collisionless components at the time of best match in the simulation. Following Belokurov et al. (2014), the coordinates of the stream particles are projected on the debris plane defined by the pole located at $\left(l_{\mathrm{GC}}, b_{\mathrm{GC}}\right)=$ $\left(275^{\circ},-14^{\circ}\right)$. Prominent tidal features are clearly visible for both the dark matter and stellar particles. Shells and arcs corresponding to apocentric pile-ups appear in both the stellar and dark matter component. Interestingly, both the dark and visible particles form large-scale streams at Galactocentric radii well beyond the apocenter distances reached by the Sgr core. The fact that some of the Sgr stars end up outside of the initial orbit can be understood by analogy with the energy redistribution that occurs in tidal disruption events (TDEs). In TDEs, half of the stellar mass gains enough energy to reach the escape speed, while the rest becomes more tightly bound (see e.g. Rees 1988; Guillochon et al. 2016). The energy gained by the leading arm from the tidal force could lead to large apocentric distances. Our simulation features such extended tidal arms because the initial orbital radius of Sgr is more remote than in previous studies. We have labeled the three most prominent distant extensions $\mathrm{N}_{\mathrm{W}}, \mathrm{N}_{\mathrm{E}}$ and $\mathrm{S}$, standing for the northwestern, northeastern and southern branches, respectively. We expect the sharpness of these stellar structures to be affected by the initial distribution of stars in the Sgr model. For a disk-less progenitor, the streams are likely to be less well-defined. Currently, the most distant tidal debris identified are part of the Sgr stream trailing tail, with apocentric distances on the order of $102.5 \pm 2.5 \mathrm{kpc}$ according to Belokurov et al. (2014). Figure 6 shows that our model predicts signiticant tidal features at distances $>100 \mathrm{kpc}$.

\subsection{Comparison to Stream Data}

In Figure 9 we present a comparison between available data and the modeled stream features projected on the Sgr orbital plane as defined by Belokurov et al. (2014). In the right panel, black crosshairs show data from Sloan Digital Sky Survey (SDSS) Data Release 5 and 8 as aggregated in Figure 10 of Belokurov et al. (2014). The size of the markers are indicative of the largest distance errors present in the dataset. Our choice of initial conditions for the Sgr baryonic components likely affects the thickness of the simulated stream. We did not try to vary the velocity dispersion of the stellar particles to optimize for stream width.

Both the leading and trailing arm SDSS detections have obvious counterparts in the simulation. The leading branch of the simulated stream presents a small offset both with the observed data and the model by Law \& Majewski (2010) shown in Belokurov et al. (2014, Fig. 10): the apocenter distance appears $\sim 10 \mathrm{kpc}$ larger than the measured value of $47.8 \pm 0.5 \mathrm{kpc}$ (Belokurov et al. 2014 ), representing a $\sim 20 \%$ mismatch. The position angle of the apocenter location is also slightly different from the data. Belokurov et al. (2014) have argued that the precession angle of only $93.2^{\circ} \pm 3.5^{\circ}$ measured from the SDSS data is an indication that the MW dark matter density falls more quickly with radius than a logarithmic potential (with typical precession angles of $120^{\circ}$ ). While orbital energy and angular momentum also play a minor role, the fact that the simulated precession angle appears $\sim 10^{\circ}$ larger than the measured value might suggest a steeper model potential is needed for the MW.

However, the model's most promising feature is that it successfully reproduces the distant trailing arm of the Sgr stream. Initially detected by Newberg et al. (2003), remote Sgr stellar debris in the Northern hemisphere were later confirmed by Ruhland et al. (2011), Drake et al. (2013), Belokurov et al. (2014), Koposov et al. (2015), and Li et al. (2016). Earlier studies hesitated to assign this structure to the Sgr stream, as simulations did not show any counterparts to these distant stars (e.g. Ruhland et al. 2011; Drake et al. 2013). Importantly, our model correctly replicates both the measured apocenter distance of $102.5 \pm 2.5 \mathrm{kpc}$ and the position angle found by Belokurov et al. (2014). Two main mechanisms have been invoked in the literature to explain the difference 

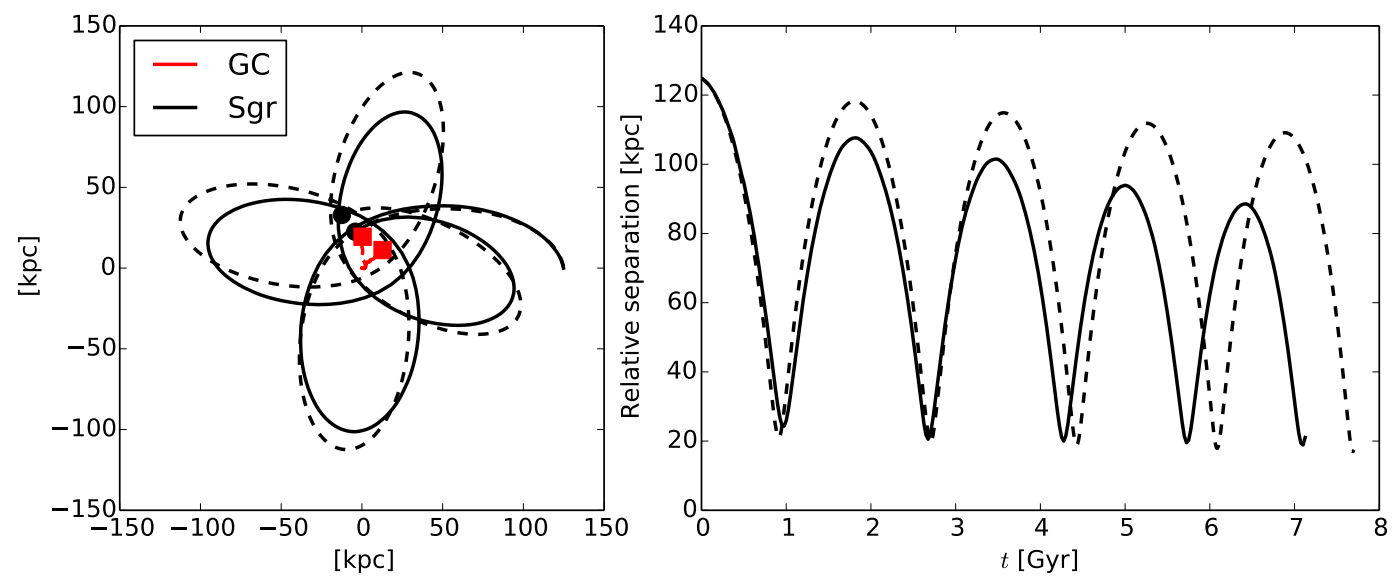

FIG. 6.- Comparison of the best-fit Sgr orbit computed with GADGET and the semi-analytic model described in Section 2 (solid and dashed lines, respectively). Left panel: trajectories of the Sgr progenitor (red) and MW Galactic Center (black) in the Sgr orbital plane. The current dynamical centers of the two galaxies are indicated by a colored dot and square, respectively. Right panel: separation between the two galaxies as a function of time since the beginning of the calculation. The closest match to the current position and velocity of Sgr is reached after 7.71 Gyr in the semi-analytic model and 7.13 Gyr in the $N$-body run.
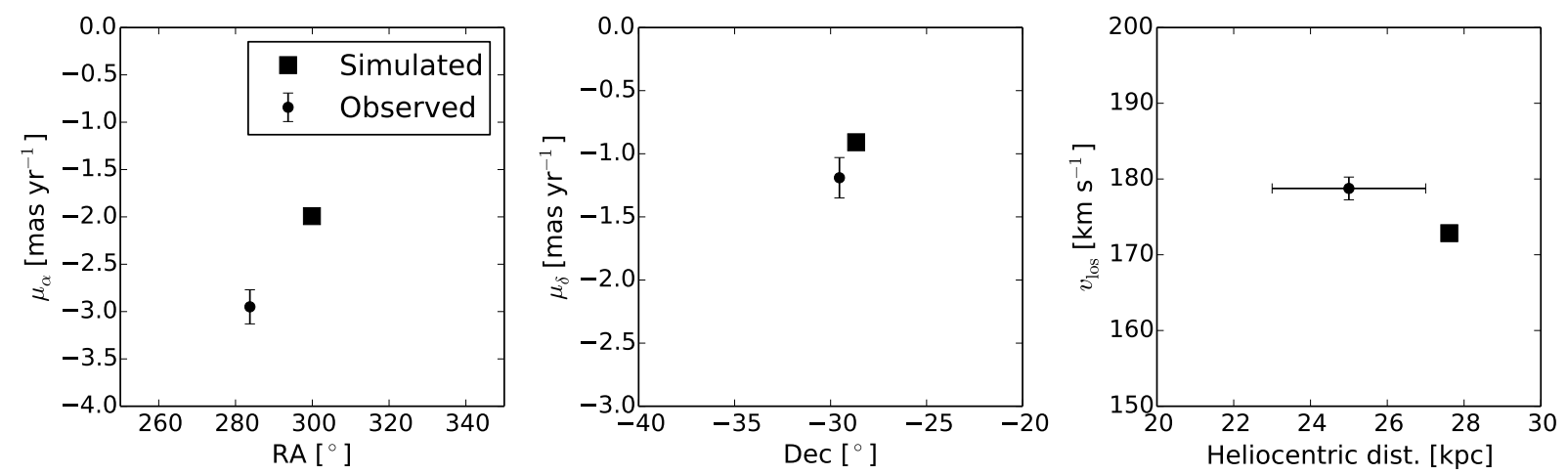

FIG. 7.- Comparison of the observed coordinates of Sgr to those simulated with an $N$-body code. As in Fig 5 , the position and velocity are shown as projected along the line of sight and in equatorial coordinates on the sky, and error bars show the $1 \sigma$ uncertainties associated with each measurement.

in apocentric distances of $\sim 50 \mathrm{kpc}$ measured by $\mathrm{Be}-$ lokurov et al. (2014). According to Chakrabarti et al. (2014), the disparity is due to dynamical friction modulating the eccentricity of the orbit of Sgr. Given the high initial separation/progenitor mass regime investigated in our simulation, dynamical friction plays an important role in shrinking down the pericenter and apocenter distances reached by the Sgr dwarf. On the other hand, Gibbons et al. (2014) find that the stream behavior depends primarily on the host potential and secondarily on the satellite progenitor properties.

Figure 10 shows the present-day distribution of the simulated Sgr stars in different projections of phasespace. The left-hand-side panels feature all Sgr star particles inside a Galactocentric radius of $100 \mathrm{kpc}$, along with stellar tracer data from the Two Micron All Sky Survey (2MASS) from Majewski et al. (2004). Overall, the simulation produces a reasonable match to the observed tidal debris characteristics shown in black squares. Unlike the stellar distribution presented in Gòmez et al. (2015), we successfully reproduce the distant branch of the leading arm observed at $\mathrm{RA} \sim 200^{\circ}$ and distances $>50 \mathrm{kpc}$. The model however predicts comparatively fewer stars at smaller distances. This comparison is lim- ited, however, by the low number of Sgr stellar particles in the simulation and the resulting relatively sparse sampling. Similarly, the simulated data at RA $\sim 150^{\circ}$ and dec $\sim 20^{\circ}$ are too coarse to distinguish the presence of the bifurcation in the leading stream detected by Belokurov et al. (2006). Observational and theoretical studies (e.g. Belokurov et al. 2014; Peñarrubia et al. 2010, 2011) have not yet reached a consensus regarding the dependence of the bifurcated tails on the morphology of the MW and the Sgr progenitor. More comprehensive simulations and detailed testing of galactic model parameters are needed in order to shed light on the origins of the stream bifurcation.

The simulated stellar locations in (RA, dec) feature a systematic offset compared to the data, and the location of highest particle density does not line up exactly with the Sgr centroid. Such coherent shifts of the simulated stream occasionally appeared across the numerous test simulations carried out for this study. The combination of coarse sampling of possible Sun locations on the Fibonacci sphere (see Section 2.2) as well as the time interval of 25 Myr between subsequent output snapshots may be responsible for these offsets. Finer sampling and tuning of the simulation parameters could perhaps remove 


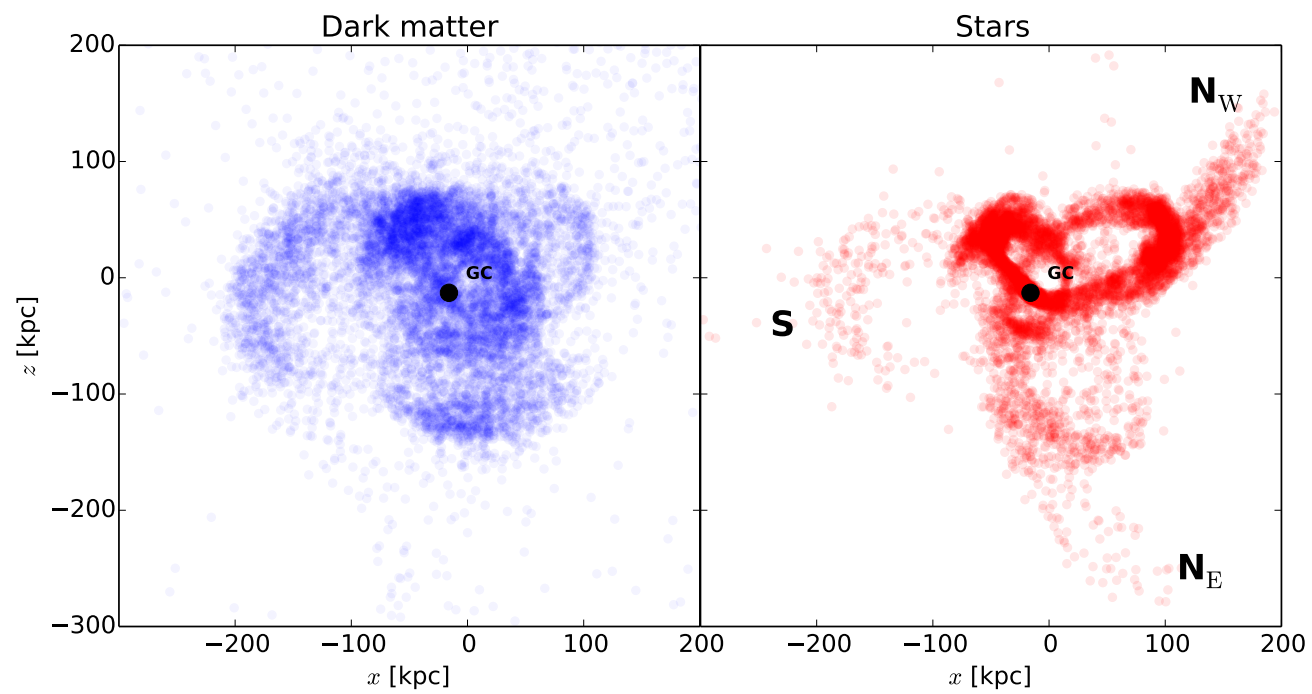

Fig. 8. - Large-scale view of the Sgr debris stream. The projection plane is defined by its pole at Galactocentric coordinates $(l, b)=$ $\left(275^{\circ},-14^{\circ}\right)$, following Belokurov et al. (2014). The locations of the Sun and MW center are marked by a black star and square, respectively. Left panel: Dark matter particles. Right panel: Stellar particles. The three most prominent distant branches of the Sgr stream are labeled $\mathrm{N}_{\mathrm{W}}$ (Northwest), $\mathrm{N}_{\mathrm{E}}$ (Northeast) and $\mathrm{S}$ (South).

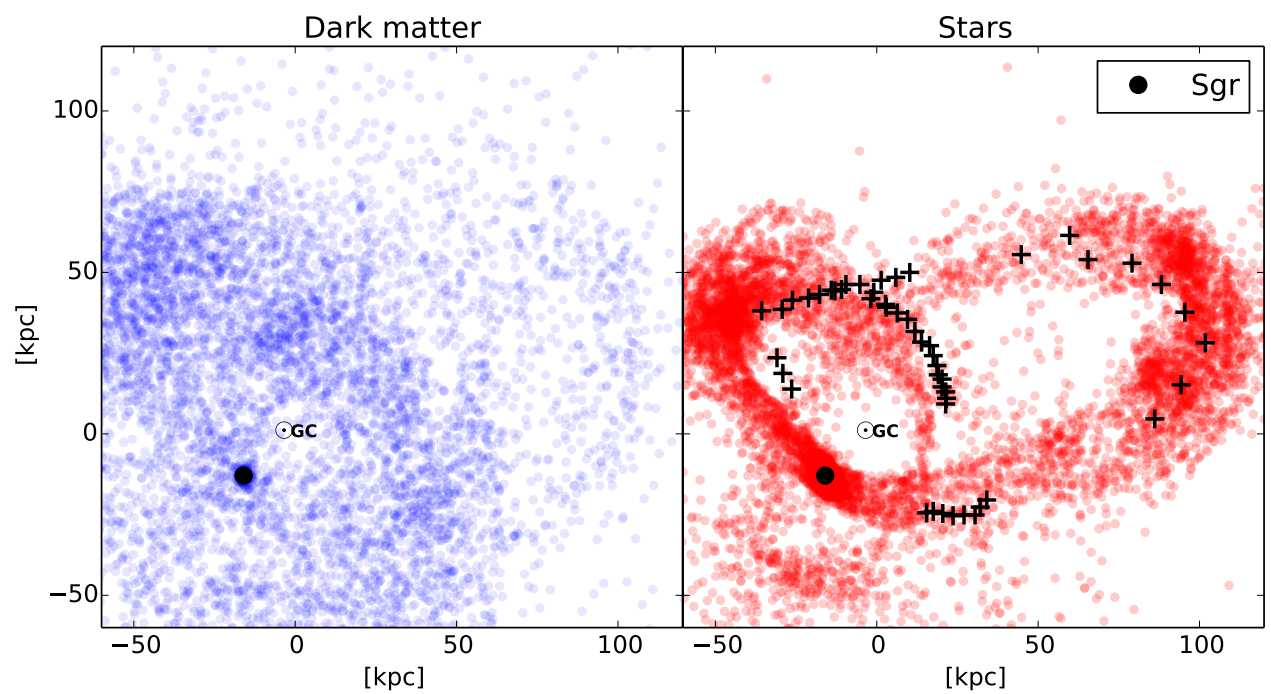

FIG. 9.- Nearby debris stream projected in the Sgr orbital plane defined by Belokurov et al. (2014) (as in Fig 8). The locations of the Sun, MW center and Sgr dwarf remnant are marked by a black star, square and circle, respectively. Left panel: Dark matter particles. Right panel: Stellar particles. The black plus signs show SDSS data from Belokurov et al. (2014) Figure 10. The model reproduces both the leading and trailing arms of the stream.

this issue. Alternatively, this angular offset may indicate that the simple spherical potential used to model the MW halo in this simulation is insufficient.

Additionally, the run of line of sight velocities for the leading $\operatorname{arm}\left(250^{\circ} \lesssim \mathrm{RA} \lesssim 150^{\circ}\right)$ features a discrepancy with the data similar to that discussed in other studies (e.g. Helmi 2004, Law et al. 2005). Helmi (2004) suggest that a prolate model of the MW potential is required to solve this mismatch. However, Law et al. (2005) and Johnston et al. (2005) point out that such models introduce a discordance with the precession angles measured from stream M-giants. While these studies disagree on the exact nature of the MW triaxiality (oblate vs. prolate), there is a consensus that it is difficult to reproduce the leading arm radial velocities with a simple spherically symmetric model (such as the one used in our study). On the other hand, some papers (Law \& Majewski 2010, Gòmez et al. 2015) have shown that the LMC can introduce significant perturbations to the phase-space distribution of Sgr debris. These hypotheses could be tested in future iterations of the orbital model presented in our study.

The set of panels on the right of Figure 10 shows the predicted distribution of stars beyond distances of $100 \mathrm{kpc}$, where no Sgr stream debris have yet been identified with certainty. The large-scale tidal features labeled in Figure 8 appear as prominent clouds of stars in these panels. The structures located at $\mathrm{RA} \sim 100^{\circ}, \sim 5^{\circ}$, and 

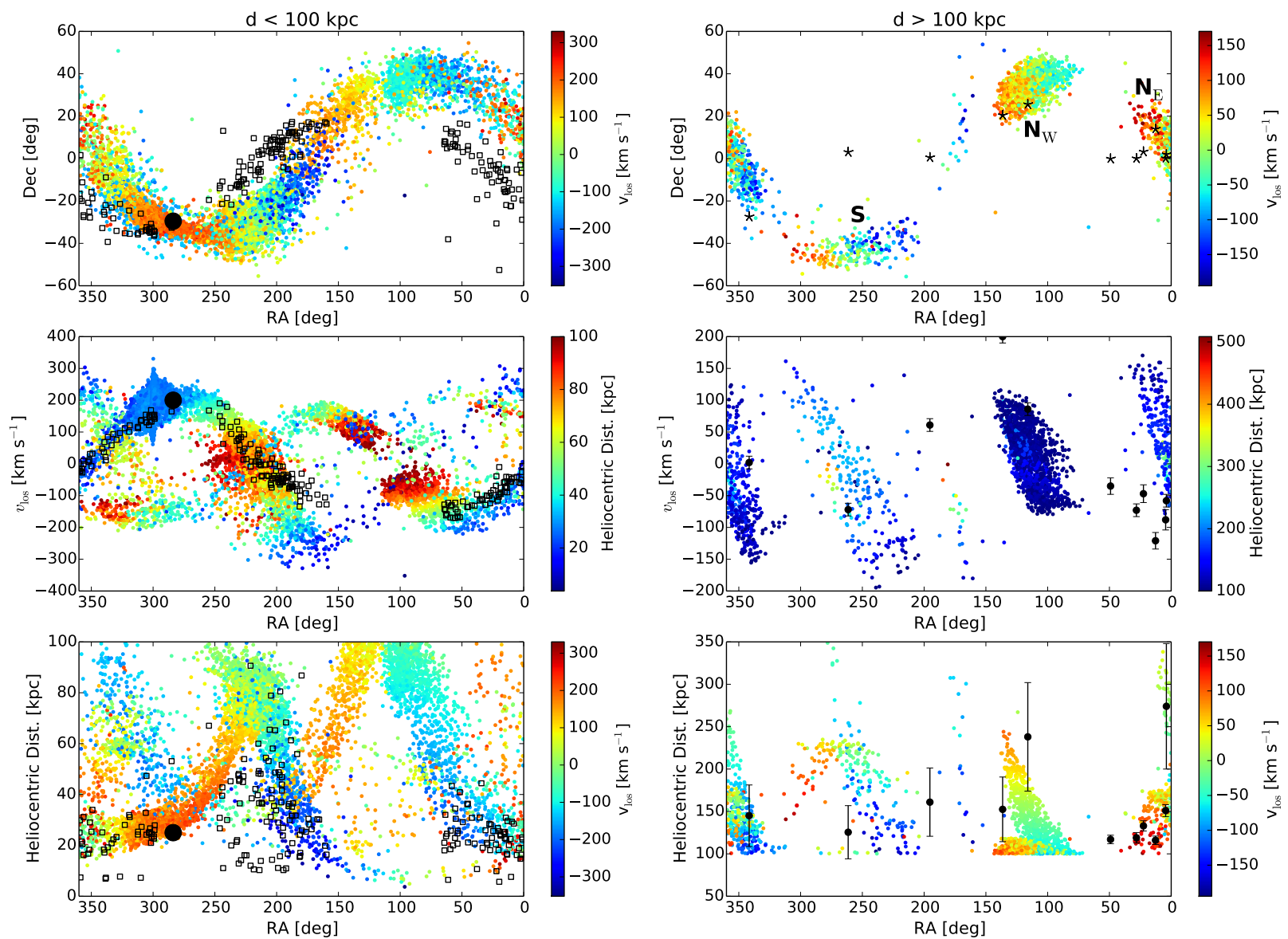

FIG. 10.- Equatorial coordinates, heliocentric distance and Galactic Standard of Rest line-of-sight velocity of the Sgr stellar particles at the present time. The simulated stars are color coded according to distance or line-of-sight velocity, as indicated in the color bars. The observed location of the Sgr remnant core is marked by a black circle. Left panels: Stellar particles with distances $<100$ kpc. Black squares show data for M-giant stars from the Two Micron All Sky Survey (2MASS) from Majewski et al. (2004). We have chosen axis ranges identical to those in Gòmez et al. (2015) (Figure 8) to allow direct comparison with the N-body model used in their study, which is based on the work of Purcell et al. (2011). Right panels: Stellar particles with distances $>100 \mathrm{kpc}$. The features located at RA $\sim 100^{\circ}$, $\sim 5^{\circ}$, and $\sim 250^{\circ}$ correspond to the distant $\mathrm{N}_{\mathrm{W}}, \mathrm{N}_{\mathrm{E}}$, and $\mathrm{S}$ branches, respectively. The distant stars detected by Deason et al. (2012a) and Bochanski et al. (2014b) are displayed as black markers. Note the smaller range of line-of-sight velocities for these distant stars, captured closer to orbital turnaround. Of the 11 distant detections plotted here, approximately half coincide with the predicted Sgr stream structure. 
$\sim 250^{\circ}$ correspond to those labeled $\mathrm{N}_{\mathrm{W}}, \mathrm{N}_{\mathrm{E}}$, and $\mathrm{S}$ in Figure 8, respectively. These distant branches can be distinguished from the closer debris in that area of the sky through line-of-sight velocities (color-coded with a different range for the distant stars), which are generally lower by $\sim 50 \mathrm{~km} \mathrm{~s}^{-1}$ since they approach turnaround.

While no tidal streams were so far identified at such large distances (Drake et al. 2013), Figure 10 includes the parameters of the few MW stars found at distances $>100 \mathrm{kpc}$. A variety of stellar tracers have been used to map the inner halo. However, due to faint limits on the order of $r \lesssim 21$, surveys so far have only yielded a dozen or so stars beyond $100 \mathrm{kpc}$. Deason et al. (2012a) provide a sample of distant blue horizontal branch and N-type carbon stars, later complemented by the M-giants detected by Bochanski et al. (2014b). In their UKIRT Infrared Deep Sky Survey (UKIDSS) data, Bochanski et al. (2014b) were able to identify two stars with distances above $200 \mathrm{kpc}$, making them the most distant known MW stars. Figure 10 shows that these most distant stars appear consistent with the remote northern stream branch predicted in our simulation. Bochanski et al. (2014b) already associated the M-giants in a distance range of 20-90 kpc in the UKIDSS data with Sgr. However, the more distant detections in their sample were not connected with the stream because the models of Law \& Majewski (2010) do not feature stars beyond $\sim 75 \mathrm{kpc}$. Our study shows that some of the most distant known MW stars could have originated in Sgr.

\section{DISCUSSION AND CONCLUSIONS}

We have taken a two-pronged approach to building a new model of the Sgr orbit through the MW halo. Rather than integrate the present-day phase-space coordinates backwards in time, we perform an exploration of parameter space with a semi-analytic integration of the Sgr trajectory starting 7-8 Gyr ago. This method is chosen because it allows for the inclusion of non time-reversible effects. In particular, dynamical friction and tidal stripping are expected to be important in the regime where the mass of the Sgr progenitor is $\gtrsim 10^{10} \mathrm{M}_{\odot}$. We then build on these results to simulate the trajectory of Sgr with GADGET and compare the resulting tidal stream to observational data. Our main conclusions are as follows:

1. Comparing the simulated position and velocity of Sgr today to the measured quantities, we find that our analytic model favors a narrow range of the initial orbital angular momentum of Sgr, with large incidence angles $\left(\theta_{\text {init }} \gtrsim 60^{\circ}\right)$ and initial velocities in the range $80 \mathrm{~km} \mathrm{~s}^{-1} \lesssim v_{\text {init }} \lesssim 100 \mathrm{~km} \mathrm{~s}^{-1}$.

2. The mock Sgr stream resulting from the GADGET simulation reproduces most of the 2MASS (Majewski et al. 2004) sky positions, heliocentric distances, and line of sight velocities. Similarly to previous studies, the leading arm line of sight velocities are not replicated by the model, suggesting that further work on the triaxiality of the MW halo or the inclusion of the LMC influence is necessary.

3. The simulated debris stream projected on the plane of the Sgr orbit is in unprecedentedly good agreement with the SDSS stellar tracers from Belokurov et al. (2014). The stream apocentric distances and position angles are reproduced to within $20 \%$ of the measured values. In particular, the model of the Sgr orbit presented here is the first to naturally reproduce the recently detected distant trailing arm at $\sim 100 \mathrm{kpc}$, optimizing only for the remnant coordinates rather than fitting for the stream properties. We believe that this feature arises because of the larger initial separation $(125 \mathrm{kpc})$ used in our simulation compared to other works in the literature (e.g. Law \& Majewski 2010, Purcell et al. 2011, Gòmez et al. 2015).

4. Above all, this work predicts the existence of two novel and distant arms of the Sgr stream. Currently the most distant SDSS stream detections are located at distances of $\sim 102.5 \pm 2.5 \mathrm{kpc}$ (Belokurov et al. 2014). The simulation presented here includes stellar overdensities at distances of up to $\sim 250-300 \mathrm{kpc}$, extending beyond the MW virial radius. We provide their predicted positions on the sky, heliocentric distances and line of sight velocities for possible future observational searches. The most distant known stars of the MW coincide with our predicted streams in both position and small radial velocities. If verified observationally, the distant branches of the Sgr stream would be the farthest-ranging stellar stream in the MW halo known to date.

UKIDSS is sensitive to M-giants beyond the MW virial radius and its distant detections appear consistent with our predicted stream. Further findings within existing datasets may be possible with the Panoramic Survey Telescope \& Rapid Response System ${ }^{2}$ (Pan-STARRS) forced photometry method, or with the Dark Energy Camera Legacy Survey ${ }^{3}$ (DECaLS). In the future, the high depth of Large Synoptic Survey Telescop $\AA^{4}$ (LSST) data will allow detailed mapping of the outer halo in the visible band, while the Wide Field Infrared Survey Telescop $\AA^{5}$ (WFIRST) will improve on current UKIDSS photometry in the infrared. The detection and characterization of the distant branches of the Sgr stream would provide an unprecedented opportunity to probe the outer envelope of the MW, which is also influenced by the neighboring Andromeda galaxy. The Gaia mission will accurately map a large volume of the MW at smaller Galactocentric radii. With a complete picture of the MW mass distribution from the solar neighborhood to the outskirts of the halo, we will be able to place our Galaxy and the Local Group in a cosmological context.

We thank Laura Blecha, Vasily Belokurov, Gurtina Besla and Facundo Gòmez for valuable discussions, as well as the referee for helpful comments on the manuscript.

\footnotetext{
${ }^{2}$ http://pan-starrs.ifa.hawaii.edu/public/

3 http://legacysurvey.org/decamls/

${ }^{4}$ https://www.lsst.org

${ }^{5}$ http://wfirst.gsfc.nasa.gov
} 


\section{REFERENCES}

Barkana R., Loeb A. 2001, Phys. Rep., 349, 125

Behroozi, P. S., Conroy, C., \& Wechsler, R. H. 2010, ApJ, 717, 379

Bellazzini, M., Correnti, M., Ferraro, F. R., et al. 2006, A\&A, 446, L1

Bellazzini, M., Ibata, R. A., \& Chapman, S. C. 2008, AJ, 136, 1147

Belokurov, V., Zucker, D. B., Evans, N. W., et al. 2006, ApJ, 642 , L137

Belokurov, V., Koposov, S. E., Evans, N. W., et al. 2014, MNRAS, 437, 116

Besla, G., Kallivayalil, N., Hernquist, L., et al. 2007, ApJ, 668, 949

Bochanski, J. J., Willman, B., Caldwell, N., et al. 2014b, ApJ, 790, L5

Binney, J. \& Tremaine, S. 1987, Galactic Dynamics (Princeton: Princeton Univ. Press), 747

Chakrabarti S., Quillen A., Chang P., Merritt D., 2014, preprint (arXiv:e-prints)

Conroy, C., \& Wechsler, R. 2009, ApJ, 696, 620

Deason, A. J., Belokurov, V., Evans, N. W., et al. 2012a, MNRAS, 425, 2840

Deg, N., \& Widrow, L. 2013, MNRAS, 428, 912

de la Rosa, I. G., La Barbera, F., Ferreras, I., et al. 2016, MNRAS, 457, 1916

Diemer, B., \& Kravtsov, A. V. 2015, ApJ, 799, 108

Dierickx, M., Blecha, L., \& Loeb, A. 2014, ApJL, 788, L38

Dinescu, D. I., Girard, T. M., van Altena, W. F., \& López, C. E. 2005, ApJ, 618, L25

Drake, A. J., Catelan, M., Djorgovski, S. G. et al. 2013, ApJ, 765, 154

Fakhouri, O., Ma, C-P., \& Boylan-Kolchin, M. 2010, MNRAS, 406, 2267

Fellhauer, M., Belokurov, V., Evans, N. W., et al. 2006, ApJ, 651, 167

Gao, L., Loeb, A., Peebles, P. J. E., et al. 2004, ApJ, 614, 17

Gibbons, S. L. J, Belokurov, V., \& Evans, N. W. 2014, MNRAS, 445,3788

Gibbons, S. L. J., Belokurov, V., Evans, N. W. 2016, preprint, (arXiv:1607.00803)

Gòmez, F. A., Besla, G., Carpintero, D. D., et al. 2015, ApJ, 802, 128

Gonzàlez, A. 2010. Math Geosci. 42, 49

Guillochon, J., McCourt, M., Chen, X., et al. 2016, ApJ, 822, 48

Hammer, F., Puech, M., Chemin, L., Flores, H., \& Lehnert, M. D. 2007, ApJ, 662, 322

Hashimoto, Y., Funato, Y., \& Makino, J. 2003, ApJ, 582, 196

Helmi, A. 2004, ApJ, 610, L97

Hernquist, L. 1990, ApJ, 356, 359

Honma, M., Nagayama, T., Ando, K., et al. 2012, PASJ, 64, 136

Ibata, R. A., Gilmore, G., \& Irwin, M. J. 1994, Nature, 370, 194

Ibata, R., Lewis, G. F., Irwin, M., et al. 2001, ApJ, 551, 294

Ibata, R., Lewis, G. F., Martin, N. F., et al. 2013, ApJ, 765, L15

Jiang, I-G., \& Binney, J. 2000, MNRAS, 314, 468

Johnson, D. R. H., \& Soderblom, D. R. 1987, AJ, 93, 864

Johnston, K. V., Spergel, D. N., \& Hernquist, L. 1995, ApJ, 451, 598

Johnston, K. V., Law, D. R., \& Majewski, S. R. 2005, ApJ, 619, 800

Kallivayalil, N., van der Marel, R. P., Besla, G., et al. 2013, ApJ, 764,161
Keselman, J. A., Nusser, A., \& Peebles, P. J. E. 2009, Phys. Rev. D, 80, 063517

King, I. 1962, AJ, 67, 471

Koposov, S. E., Belokurov, V., Zucker, D. B., et al. 2015, MNRAS, 446, 3110

Kunder, A., \& Chaboyer, B. 2009, AJ, 137, 4478

Law, D. R., Johnston, K. V., \& Majewski, S. R. 2005, ApJ, 619, 807

Law, D. R., Majewski, S. R., \& Johnston, K. V. 2009, ApJ, 703, L67

Law, D. R., \& Majewski, S. R. 2010, ApJ, 714, 229

Li, J., Smith, M. C., Zhong, J., et al. 2016, ApJ, 823, 59

Loeb, A., \& Peebles, P. J. E. 2003, ApJ, 589, 29

Lu, Y., Benson, A., Mao, Y-Y., et al. 2016, ApJ, submitted

Majewski, S. R., Skrutskie, M. F., Weinberg, M. D., \& Ostheimer, J. C. 2003, ApJ, 599, 1082

Majewski, S. R., Kunkel, W. E., Law, D. R., et al. 2004, AJ, 128, 245

Massari, D., Bellini, F. R., van der Marel, R. P., et al. 2013, ApJ, 779,81

Mollitor, P., Nezri, E., \& Teyssier, R. 2015, MNRAS, 447, 1353

Navarro, J. F., Frenk, C. S., \& White, S. 1997, ApJ, 490, 493

Newberg, H. J., Yanny, B., Grebel, E. K., et al. 2003, ApJ, 596, L191

Niederste-Ostholt, M., Belokurov, V., Evans, N. W., \& Peñarrubia, J. 2010, ApJ, 712, 516

Peñarrubia, J., Benson, A. J., Martínez-Delgado, D., \& Rix, H. W. 2006, ApJ, 645, 240

Peñarrubia, J., Belokurov, V., Evans, N. W., et al. 2010, ApJL, 408, L26

Peñarrubia, J., Zucker, D. B., Irwin, M. J., et al. 2011, ApJL, 727, L2

Planck Collaboration, 2015, arXiv:1502.01589

Price-Whelan, A. M., \& Johnston, K. V. 2013, ApJ, 778, L12

Pryor, C., Piatek, S., \& Olszewski, E. W. 2010, ApJ, 139, 839

Purcell, C. W., Bullock, J. S., Tollerud, E. J., et al. 2011, Nature, 477,301

Purcell, C. W., Zentner, A. R., \& Wang, M-Y 2012, JACP, 8, 27

Rees, M. J. 1988, Nature, 333, 523

Reid, M. J., Menten, K. M., Brunthaler, A., et al. 2014, ApJ, 783, 130

Ruhland, C., Bell, E. F., Rix, H.-W., \& Xue, X.-X. 2011, ApJ, 731,119

Schönrich, R., Binney, J., \& Dehnen, W. 2010, MNRAS, 403, 1829

Sohn S. T., et al. 2015

Springel, V. 2005, MNRAS, 364, 1105

Torrey, P., Wellons, S., Machado, F., et al. 2015, MNRAS, 454, 2770

van der Marel, R. P., Fardal, M., Besla, G., et al. 2012, ApJ, 753, 8

van der Marel, R. P., Besla, G., Cox, T. J., et al. 2012, ApJ, 753, 9

Vera-Ciro, C., \& Helmi, A. 2013, ApJ, 773, L4

Wellons, S., Torrey, P., Ma, C.-P., et al. 2016, MNRAS, 456, 1030

Wyse, R. F. G. 2001, in ASP Conf. Ser. 230, Galaxy Disks and Disk Galaxies, ed. J. G. Funes \& E. M. Corsini (San Francisco, CA: ASP), 71

Xue, X. X., Rix, H. W., Zhao, G., et al. 2008, ApJ, 684, 1143 